\title{
PROBABILITY OF TRYPANOSOMA CRUZI TRANSMISSION BY TRIATOMA INFESTANS (HEMIPTERA: REDUVIIDAE) TO THE OPOSSUM DIDELPHIS ALBIVENTRIS (MARSUPIALIA: DIDELPHIDAE)
}

\author{
J. RABINOVICH, N. SCHWEIGMANN, V. YOHAI, AND C. WISNIVESKY-COLLI \\ Departamento de Ciencias Biológicas y Departamento de Matemáticas, Facultad de Ciencias Exactas y Naturales, \\ Universidad de Buenos Aires, Ciudad Universitaria, Núnez, 1428 Buenos Aires, Argentina
}

\begin{abstract}
The probability of Trypanosoma cruzi transmission to opossums by independent events of predation and fecal contamination during feeding ("biting") with positive Triatoma infestans was estimated. Negative female opossums were challenged for $23 \mathrm{hr}$ with 10 infected third and fourth instars of $T$. infestans, and tests for positivity for $T$. cruzi by xenodiagnosis were performed at 30,60, and 90 days. From these data, seven probability parameters were estimated by maximum likelihood, and likelihood ratio statistics confidence intervals were calculated. Simultaneous estimation of $p_{1}$ (probability that a "bite" will infect an opossum), $p_{3}$ (probability that a bug that has been eaten by an opossum will infect it), and $p_{6}$ (probability that the opossum will become infected if faced with an infected triatomine), resulted in $\hat{p}_{1}=0.06, \hat{p}_{3}=0.075$, and $\hat{p}_{6}=0.059$. On average, each opossum should be exposed to an average of 700 encounters with bugs during its life, resulting in about eight potentially infective contacts, to produce the $35 \%$ opossum prevalence found in the field.
\end{abstract}

\section{INTRODUCTION}

Triatomines feed on a variety of vertebrate hosts, with opossums being a frequent one. In all countries where the role of opossums as reservoirs of Trypanosoma cruzi has been studied, a relatively high prevalence of $20-100 \%$ was found. ${ }^{1-8}$ In Argentina, Didelphis albiventris is the most important wild reservoir of $T$. cruzi, with an annual prevalence in Santiago del Estero that varied between $29 \%$ and $50 \%$ (Schweigmann N, unpublished data). This prevalence of this host coexists with a very low triatomine (particularly for Triatoma guasayana) infection, sometimes less than $1 \% .^{6,10}$ Near 40 years ago, the dilemma of how such a high opossum T. cruzi infection rates could be maintained with the observed low infection rates of triatomines was posed by McKeever and others ${ }^{11}$ and since then remains unanswered. Opossums are known to become infected with $T$. cruzi after being "bitten" by and/or ingesting triatomines. ${ }^{12-14}$ When triatomines "bite" to feed on a host, the biting itself does not inoculate the parasite. Transmission takes place by fecal contamination during or after feeding. To simplify the writing, we will refer to this transmission process as "biting". These trophic associations between triatomine vectors and opossums occur in their shelters, implying they are a possible focus of parasite transmission. In other trypanosomatids, such as African trypanosomes and leishmanias, the feeding behavior of infected vectors is modified by the parasite, thus increasing the frequency and duration of "biting," 15 leading to high prevalence in mammalian hosts associated with low infection rates of vectors. However, in the case of triatomines, the presence of $T$. cruzi does not affect the feeding behavior of infected insects. ${ }^{16}$ Thus, an alternative explanation of the high opossum prevalence resulting from few infected vectors should be investigated at the level of probability of transmission from infected triatomines to opossums. In a laboratory device that simulated a natural opossum burrow, in which different opossums were independently challenged for $23 \mathrm{hr}$ with 10 infected $T$. infestans, $29 \%$ of the opossums became infected $(40 \%$ among the opossums that ingested triatomines and $21 \%$ among the ones that did not ingest triatomines). Experimental opossum in- fections in the laboratory resulted in prevalences similar to those observed in the field, ${ }^{14}$ suggesting that for adult $D$. albiventris, a few infective contacts with infected triatomines might be sufficient to produce infection. Males are found infected in nature less frequently than females, and very rarely become infected experimentally (Schweigmann N, unpublished data). Also, since females have a much longer longevity than males, they are the main reservoirs of $T$. cruzi in the wild. To evaluate the possible relevance of opossums in the transmission of $T$. cruzi to humans, we used only female data. In this report, we estimate the probability of $T$. cruzi infection of female opossums when exposed to infected Triatoma infestans.

\section{MATERIALS AND METHODS}

Sources of data. We used the original data of the experiment carried out by Schweigmann and others. ${ }^{14}$ Although details can be found therein, what follows is a basic summary of the methods used. Wild captured, noninfected $D$. albiventris were challenged for $23 \mathrm{hr}$ with infected T. infestans. The experiment consisted of 13 replicates, each with one female opossum and a random mixture of 10 third and fourth instar triatomines. ${ }^{14}$ The device used simulated a natural opossum burrow: a hardboard cage $(50 \times 50 \times 45 \mathrm{~cm})$ with one of the walls containing a removable wooden panel that acted as a shelter for triatomines, a fine wire net window for ventilation, and sand on the floor to absorb moisture. Opossums were starved for 1-2 days, and infected triatomines were fasted for 20-30 days before the trials. After 23 $\mathrm{hr}$, the number and nutritional status of the bugs were recorded; missing bugs were considered ingested by the opossums. For a three-month period after the trials the opossums were tested every 30 days for positivity to $T$. cruzi by xenodiagnosis.

Statistical analysis. The relevant experimental variables are identified as $X$ : the number of bugs that have "bitten" the opossum, $W$ : the number of bugs that have been eaten by the opossum, and $Y$ : a variable that takes the value 1 if the opossum becomes infected, and 0 if it does not. If $n$ 
trials are carried out, each with different opossums and bugs, the results of the observations in each cage $(i)$ will be $\left(x_{i}\right.$, $\left.w_{i}, \mathrm{y}_{i}\right)$, where $1 \leq i \leq n$; in our study $n=13$. Let us call $p_{1}$ the probability that a "bite" will infect an opossum. Let us call $p_{2}$ the probability that a bug that has been eaten by an opossum will infect it, either because it had "bitten" the opossum previously or as a direct result of having been ingested. The parameters $p_{1}$ y $p_{2}$ are unknown and will have to be estimated from the sample of $n$ experiments.

Based on the assumption that the transmission of T. cruzi from the bugs to the opossums is independent, the probability function of $Y$ conditional $(X, W)$ for a given trial as a function of $p_{1}$ and $p_{2}$ will be given by

$P\left(Y=0 \mid X=x, W=w, p_{1}, p_{2}\right)=\left(1-p_{1}\right)^{x}\left(1-p_{2}\right)^{w}$

which results from the fact that for the opossum to remain non-infected neither the "bites" nor the ingestions should produce infection. It then follows that

$$
\begin{aligned}
P\left(Y=1 \mid X=x, W=w, p_{1}, p_{2}\right) \\
\quad=1-P\left(Y=0 \mid X=x, W=w, p_{1}, p_{2}\right) \\
\quad=1-\left(1-p_{1}\right)^{x}\left(1-p_{2}\right)^{w}
\end{aligned}
$$

The joint likelihood function of the values $y_{1}, \ldots, y_{n}$ conditional to $x_{1}, \ldots, x_{n}$ and $w_{1}, \ldots, w_{n}$ is given by

$$
L\left(p_{1}, p_{2}\right)=\prod_{i=1}^{n} P\left(Y=y_{i} \mid X=x_{i}, W=w_{i}, p_{1}, p_{2}\right)
$$

The maximum likelihood estimators $p_{1}$ and $p_{2}$ as given in (3) are obtained by maximizing $L\left(p_{1}, p_{2}\right)$. Another parameter is $p_{3}$, defined as the probability that if a bug is ingested it will infect the opossum exclusively because of the ingestion itself. However, as from the experimental results the proportion of the ingested bugs that had "bitten" the opossum before being ingested is not known, we cannot estimate $p_{3}$ without resorting to supplementary hypotheses. That is, to estimate $p_{3}$ we would need to know the probability $p_{4}$, defined as the probability that an ingested bug might had "bitten" the opossum before being eaten. In that case we can estimate $p_{3}$ as (for proof, see Appendix 1)

$$
p_{3}=\frac{p_{2}-p_{1} p_{4}}{1-p_{1} p_{4}}
$$

where the value of $p_{3}$ will be estimated using three hypotheses: i) that $p_{4}=0$ (no bug had "bitten" before being ingested), ii) that $p_{4}=1$ (all bugs had "bitten" before being ingested), and iii) that the probability that an ingested bug had "bitten" the opossum previously is the same as the probability of "biting" an opossum by non ingested bugs. If we resort to hypothesis iii we have to estimate simultaneously $p_{1}, p_{2}$, and $p_{3}$ by maximum the likelihood of $\left(x_{1}, y_{1}\right)$, $\ldots,\left(x_{n} y_{n}\right)$ conditionally to $\left(w_{1}, \ldots, w_{n}\right)$. Then we have to maximize $L^{*}\left(p_{1}, p_{3}, p_{4}\right)$ defined by

$$
\begin{aligned}
L^{*} & \left(p_{1}, p_{2}, p_{3}\right) \\
\quad & =\prod_{i=1}^{n} P\left(Y=y_{i}, X=x_{i} \mid W=w_{i}, p_{1}, p_{3}, p_{4}\right)
\end{aligned}
$$

The derivation of $L^{*}\left(p_{1}, p_{3}, p_{4}\right)$ is given in Appendix 2 .

We derive confidence intervals for $p_{1}, p_{2}$, and $p_{3}$ based on the likelihood ratio statistics (LRS). For $p_{3}$ the interval was derived assuming the hypothesis iii. For $p_{1}$ we calculated exact and approximate intervals. The exact interval requires computing the exact distribution of the LRS for all possible values of the parameters $p_{1}, p_{2}$. This was done by Monte Carlo analysis. The approximate intervals were obtained using the asymptotic distribution of the LRS, which is $\chi^{2}$ with one degree of freedom. For $p_{2}$ and $p_{3}$ we calculated only approximate intervals. Details on how these intervals are defined are given in Appendix 3.

It was also of interest to estimate the parameter $p_{6}$, defined as the probability that, exposed for $23 \mathrm{hr}$ to one infected triatomine, the opossum will become infected. We again have to assume that the "biting" of the triatomine on the opossum and the ingestion of the triatomine by the opossum are independent events. Also shown in Appendix 1 the value of $p_{6}$ is determined by

$$
p_{6}=1-\left(1-p_{1} p_{4}{ }^{\prime}\right)\left(1-p_{3} p_{5}\right)
$$

where, $p_{1}$ and $p_{3}$ are as defined before, $p_{5}$ is the probability that, faced with a triatomine for $23 \mathrm{hr}$, the opossum will eat it and $p_{4}{ }^{\prime}$ is the probability that, faced with a triatomine for $23 \mathrm{hr}$, the opossum will be "bitten" by it (it was called $p_{4}$ ' because under hypothesis iii it results in $p_{4}{ }^{\prime}=p_{4}$ ); both values were estimated from the average proportion of triatomines that were eaten by or had "bitten" on the opossums in the laboratory experiments, respectively.

Estimation of the values of $p_{4}, p_{5}$, and $p_{6}$ allow for a rough estimate of the potential number of infective encounters (contacts) between opossums and triatomines that should occur in the wild to produce an opossum prevalence similar to the one found in the field. In Appendix 4, proof is given that if a) waiting times between "bites" and ingestions follow an exponential distribution with parameters $\lambda_{x}$ and $\lambda_{w}$, respectively, and $b$ ) the average number of triatomines that an opossum will be faced with during its entire life is a random process described by the Poisson distribution with mean $\mu$, then the average proportion of triatomines that "bite" and that are ingested in an encounter, is given by

$$
\begin{gathered}
p_{4}{ }^{\prime}=1-e^{-T_{e} \lambda_{x}} \\
p_{5}=1-e^{-T_{e} \lambda_{w}}
\end{gathered}
$$

where $\lambda_{x}$ and $\lambda_{w}$ are the average "biting" and ingestion times, respectively, and $T_{e}$ is the time an encounter lasts. If the probability that an opossum becomes infected during its entire life is estimated by the field opossum prevalence $\left(p_{7}\right)$, then Appendix 4 also demonstrates that the average number of encounters between an infected triatomine and an opossum should be

$$
\mu=-\frac{\ln \left(1-p_{7}\right)}{p_{6}}
$$

Table 1 summarizes the definitions and use of the different symbols

\section{RESULTS}

Table 2 shows the experimental results in terms of the variables $X, W$, and $Y$. A contingency test using the "biting" and predation frequencies of Table 2 showed that the "bit- 
TABLE 1

Definition of symbols used*

\begin{tabular}{|c|c|}
\hline$X$ & Number of bugs that have "bitten" the opossum \\
\hline$W$ & $\begin{array}{l}\text { Number of bugs that have been eaten by the } \\
\text { opossum }\end{array}$ \\
\hline$Y$ & $\begin{array}{l}\text { Variable that takes the value } 1 \text { if the opossum } \\
\text { becomes infected, and } 0 \text { if it does not }\end{array}$ \\
\hline$n$ & $\begin{array}{l}\text { Number of trials are carried out, each with dif- } \\
\text { ferent opossums and bugs }(n=13)\end{array}$ \\
\hline$i$ & Trial number, with $1 I n$ \\
\hline$x_{i}, w_{i}, y_{i}$ & $\begin{array}{l}\text { Results of observations of variable } X, W, Y \text { in } \\
\text { cage } i\end{array}$ \\
\hline$P_{1}$ & Probability that a "bite" will infect an opossum \\
\hline$p_{2}$ & $\begin{array}{l}\text { Probability that a bug that has been eaten by an } \\
\text { opossum will infect it, either because it had } \\
\text { "bitten" the opossum previously, or as a di- } \\
\text { rect result of having been ingested }\end{array}$ \\
\hline$p_{3}$ & $\begin{array}{l}\text { Probability that if a bug is ingested it will infect } \\
\text { the opossum exclusively because of the inges- } \\
\text { tion itself }\end{array}$ \\
\hline$p_{4}$ & $\begin{array}{l}\text { Probability that an ingested bug might have } \\
\text { "bitten" the opossum before being eaten }\end{array}$ \\
\hline$p_{5}$ & $\begin{array}{l}\text { Probability that, faced with a triatomine for } 23 \\
\text { hr, the opossum will eat it }\end{array}$ \\
\hline$p_{6}$ & $\begin{array}{l}\text { Probability that, exposed for } 23 \mathrm{hr} \text { to one infect- } \\
\text { ed triatomine, the opossum will become in- } \\
\text { fected }\end{array}$ \\
\hline
\end{tabular}

ing" and predation processes are independent $\left(\chi_{1,0.05}^{2}=\right.$ $0.304, \mathrm{p}=0.5814)$. The maximum likelihood estimators of $p_{1}$ and $p_{2}$ derived from equation 3 are 0.060 and 0.112 , respectively. The estimation of $p_{3}$ resulted in different values depending on the three hypotheses about $p_{4}$; for hypothesis $i$, applying $p_{4}=0$ to equation 4 results in $p_{3}=p_{2}=0.112$; for hypothesis ii, applying $p_{4}=1$ to equation 4 results in $p_{3}$ $=0.055$. Using hypothesis iii, the simultaneous estimation of $p_{1}, p_{3}$, and $p_{4}$ after application of equation 5 resulted in $\hat{p}_{1}=0.06, \hat{p}_{3}=0.075$, and $\hat{p}_{4}=0.68$.

For $p_{1}$ we calculated confidence intervals at the 0.95 level and obtained $[0.023,0.162]$ for the exact estimate, and $[0.013,0.156]$ for the approximate estimate. Despite the small sample size $(n=13)$ the two intervals are quite similar so the $\chi^{2}$ approximation for the LRS distribution seems reasonable. In view of this result we calculated only the approximate intervals for $p_{2}$ and $p_{3}$. The interval for $p_{2}$ is $[0.016,0.288]$. The interval for $p_{3}$, assuming that hypothesis iii holds, is $[0,0.265]$. The intervals are not symmetric with respect to the point estimates because the parameters have zero as the lower bound.

The experimental results showed that $\hat{p}_{5}=0.254$ (33 of 130 triatomines were eaten, see Table 2 ). The overall probability of transmission $p_{6}$ was estimated applying this value of $\hat{p}_{5}$ to equation 7 and obtained 0.059 . Thus, in about $6 \%$ of the cases an opossum challenged with one infected triatomine for $23 \mathrm{hr}$ will become infected. We checked this result with the laboratory experiment by applying the binomial model of transmission to the situation where the number of encounters was 10 , and expressed by $1-(1-0.059)^{10}=$ 0.455 , which is quite consistent with the experimental value of 0.461 ( 6 of 13 opossums became infected; Table 2). The average "biting" and ingestion times, applying (A4-1) and (A4-2) of Appendix 4 to the laboratory results gave $\lambda_{x}=$ 0.0474 and $\lambda_{w}=0.0122$. The application of these two pa-
TABLE 2

Experimental results of challenging one female Didelphis albiventris with 10 positive Triatoma infestans bugs for $23 \mathrm{hr}$ replicated 13 times*

\begin{tabular}{cccc}
\hline $\begin{array}{c}\text { Trial number } \\
(i)\end{array}$ & $\begin{array}{c}\text { No. of } \\
\text { ingested bugs } \\
\left(w_{i}\right)\end{array}$ & $\begin{array}{c}\text { No. of bugs that have } \\
\text { "bitten" the opossum } \\
\left(\boldsymbol{x}_{i}\right)\end{array}$ & $\begin{array}{c}\text { Opossums" } \\
\text { parasitologic result } \\
\left(y_{i}\right)\end{array}$ \\
\hline 1 & 0 & 5 & 0 \\
2 & 0 & 6 & 0 \\
3 & 0 & 9 & 0 \\
4 & 0 & 10 & 0 \\
5 & 1 & 6 & 0 \\
6 & 6 & 2 & 0 \\
7 & 10 & 0 & 0 \\
8 & 0 & 8 & 1 \\
9 & 0 & 8 & 1 \\
10 & 1 & 9 & 1 \\
11 & 3 & 1 & 1 \\
12 & 6 & 1 & 1 \\
13 & 6 & 1 & 1 \\
\hline
\end{tabular}

"Parasitologic status of the opossum was determined by xenodiagnosis at 30,60 , and 90 days after the experiment. Raw data from Schweigmann and others ${ }^{14}$ is not shown in the original data therein.

rameters to equation 9 to estimate the number of triatomineopossum encounters necessary to produce an infection rate similar to the opossum prevalence in the field $(0.35)$ gave $\mu$ $=7.95$ for encounters lasting $20 \mathrm{hr}$.

\section{DISCUSSION}

The maximum likelihood estimator of $p_{1}$ shows a relatively low value of 0.060 , with a fairly wide range of possible values $[0.023,0.162]$; this implies that six of 100 "bites" by an infected triatomine will result in the infection of an opossum. This estimate of $p_{1}$ is intermediate between the maximum likelihood estimator of $p_{3}$ (transmission by predation) estimated by hypotheses ii and iii (that is, for $p_{4}$ $=1$ and $p_{4}=0.68$, respectively). We believe that the hypothesis of $p_{4}=0$ (that no bug had "bitten" before being ingested) is not very plausible for two reasons: a) the probability that a bug "bites" an opossum (among the group of non ingested bugs) is relatively high $(0.68)$, and b) hosts normally become irritated because of the bite (López W, unpublished data), increasing the bug's risk of being ingested. Thus, we believe that the real value of $p_{4}$ should lie between 0.68 and one. In that case the value of $p_{3}$ (the probability that if a bug is ingested it will infect the opossum exclusively because of the ingestion itself) would range between 0.055 and 0.075 , which is very similar to the probability that a bite will infect an opossum $(0.060)$.

Intuition would suggest a higher estimate for $p_{3}$ than for $p_{1}$ because by ingestion there is an intimate contact with the mouth mucosa that would facilitate infection, while transmission by "biting" is hindered by the mechanical barrier of a healthy skin and the density of the fur where the feces are usually deposited. However, there seems to be a compensation in both processes: the transmission by "biting" may be augmented by the licking of the opossum on the "bitten" area, increasing the parasite's possibilities of entering in direct contact with the mouth mucosa and, on the other hand, if the bugs are wholly ingested (that is, not broken down by chewing) the possibility of an intimate contact between the parasite and the mouth mucosa is reduced and, 
due to the acid environment of the digestive track, and the possibility of transmission by ingestion would be decreased. Then it seems reasonable that the values of $p_{1}$ and $p_{3}$ might be very close. This work provides the first estimates of the probability of transmission of $T$. cruzi to $D$. albiventris by the bite of an infected Triatoma infestans. Although our findings provide an apparently "low" value to the probability of transmission by "biting" $\left(p_{1}=0.06\right)$, it is about 50 times larger than the one found for humans $(0.0012)$ with the same triatomine species. ${ }^{17}$

There is no estimation on the probability of transmission by "biting" for Triatoma guasayana, the main wild insect vector in northern Argentina. Assuming a) that the average "biting" rate and the rate at which they are ingested by opossums are similar between $T$. guasayana and T. infestans, b) that in both triatomine species the "waiting times" follow an exponential distribution, and c) that an opossum remains in its shelter an average of $20 \mathrm{~h}$ each day (Schweigmann $\mathrm{V}$, unpublished data), and using the estimated values of $p_{1}$ $(0.06)$ and $p_{3}(0.075)$, each opossum should be exposed to about eight potentially infective encounters to produce a global prevalence of 35\%, as found in Santiago del Estero, Argentina. In this place $D$. albiventris lives an average of 170 days (Schweigmann V, unpublished data), and if we assume that $1 \%$ of the Triatoma guasayana are infected in the wild, ${ }^{9,10}$ solving for $\mathrm{N}$ in the binomial model of transmission $1-(1-0.059)^{0.01 \mathrm{~N}}=0.35$ (where $\left.0.059=1 / 170\right)$, we obtain $\mathrm{N}=708$. That is, each opossum should encounter an average of about 700 bugs during its life (around four bugs per day) to generate the $35 \%$ opossum prevalence found in the field. There is no indication that such a high rate of encounters in the field is plausible.

Due to the high prevalence of $T$. cruzi in wild opossum populations and its possible relevance in parasite transmission to humans, determination of the causal mechanisms involved in opossum infections are essential for Chagas disease control and/or prevention. The extremely low probability of transmission of $T$. cruzi from bugs to opossums reported here $(0.06)$ suggests three lines of future research as important in analyzing those mechanisms: 1) the determination the actual percentage of infected Triatoma guasayana in the wild, using recent techniques such as the polymerase chain reaction, 2) the assessment of the importance of the abundance and clumpiness of the distribution of Triatoma guasayana in wild biotopes used by opossums in the transmission of T. cruzi, and 3) an evaluation of the rate of visits to houses by opossums as a potential infection source of $T$. cruzi from Triatoma infestans.

Authors' addresses: J. Rabinovich, Centro de Estudios Parasitológicos y de Vectores (CEPAVE), Universidad Nacional de La Plata, Calle 2 No. 584, La Plata, 1900 Provincia de Buenos Aires, Argentina. N. Schweigmann and C. Wisnivesky-Colli, Departamento de Ciencias Biológicas, Facultad de Ciencias Exactas y Naturales, Universidad de Buenos Aires, Ciudad Universitaria, Núñez, 1428 Buenos Aires, Argentina. V. Yohai, Departamento de Matemáticas, Facultad de Ciencias Exactas y Naturales, Universidad de Buenos Aires, Ciudad Universitaria, Núñez, 1428 Buenos Aires, Argentina.

\section{REFERENCES}

1. Barretto MP, Ribeiro RD, 1979. Reservatorios silvestres do Trypanosoma cruzi. Rev Inst Adolfo Lutz 39: 25-26.
2. Fernandez AJ, Chiari E, Rodriguez RR, Dias JC, Romanha AJ, 1991. The importance of the opossum (Didelphis albiventris) as a reservoir for Trypanosoma cruzi in Bambuí, Minas Gerais State. Mem Inst Oswaldo Cruz 86: 81-85.

3. Lainson R, Shaw JJ, Fraiha H, Miles MA, Draper CC, 1979. Chagas' disease in the Amazon basin: I. Trypanosoma cruzi infections in sylvatic mammals, triatomine bugs, and man in the state of Para, North Brazil. Trans R Soc Trop Med Hyg 73: $193-204$.

4. da Rocha e Silva EO, Rehder de Andrade JC, Ribeiro de Lima A, 1975. Importancia dos animais sinantrópicos no controle da endemia chagásica. Rev Saude Publica 9: 371-381.

5. Telford SR Jr, Tonn RJ, 1982. Dinámica de Trypanosoma cruzi en poblaciones de un reservorio primario, Didelphis marsupialis, en los llanos altos de Venezuela. Bol Oficina Sanit Panam 93: 341-364.

6. Zeledón R, Solano G, Burstin L, Swartzwelder JC, 1975. Epidemiological pattern of Chagas' disease in an endemic area of Costa Rica. Am J Trop Med Hyg 24: 214-225.

7. Zeledón R, 1974. Epidemiology, modes of transmission and reservoir hosts of Chagas' disease. Trypanosomiasis and Leishmaniasis with Special Reference to Chagas' Disease. Ciba Foundation Symposium 20 (New Series). Amsterdam: Elsevier Excerpta Medica, North Holland, 51-85.

8. Pung OJ, Banks CW, Jones DN, Krissinger MW, 1995. Trypanosoma cruzi in wild raccoons, opossums, and triatomine bugs in Southeast Georgia, U.S.A. J Parasitol 81: 324-326.

9. Wisnivesky-Colli C, Schweigmann NJ, Alberti A, Pietrokovsky S, Conti O, Montoya S, Riarte A, Rivas C, 1992. Sylvatic American trypanosomiasis in Argentina. Trypanosoma cruzi infection in mammals from the Chaco Forest in Santiago del Estero. Trans R Soc Trop Med Hyg 86: 38-41.

10. Wisnivesky-Colli C, Schweigmann NJ, Pietrokovsky S, Bottazzi $\mathrm{V}$, Conti O, 1993. Peculiarities of sylvatic transmission of Trypanosoma cruzi in northern Argentina. Mem Inst Oswaldo Cruz 88 (suppl) 266: VE-35.

11. McKeever S, Gorman GW, Norman L, 1958. Occurrence of Trypanosoma cruzi-like organisms in some mammals from southwestern Georgia and northwestern Florida. $J$ Parasitol 44: 583-587.

12. Yaeger RG, 1971. Transmission of Trypanosoma cruzi infection to opossums via the oral route. J Parasitol 57: 1375-1376.

13. Ribeiro RD, Rissato García TA, Bonoma WCh, 1987. Contribuçao para o estudo dos mecanismos de transmissão do agente etiológico da doença de Chagas. Rev Saude Publica 21: 51-54.

14. Schweigmann NJ, Pietrokovsky S, Bottazzi V, Conti O, Wisnivesky-Colli C, 1995. Interactions between Didelphis albiventris and Triatoma infestans in relation to Trypanosoma cruzi transmission. Mem Inst Oswaldo Cruz 90: 679-682.

15. Lehane MJ, 1991. Biology of Blood-Sucking Insects. Hampshire, United Kingdom: Harper Collins Academic, Chapman and Hall.

16. D'Alessandro A, Mandel S, 1969. Natural infections and behavior of Trypanosoma rangeli and Trypanosoma cruzi in the vector Rhodnius prolixus in Colombia. J Parasitol 54: 846852.

17. Rabinovich JE, Wisnivesky-Colli C, Solarz ND, Gurtler RE, 1990. Probability of transmission of Chagas disease by Triatoma infestans (Hemiptera: Reduviidae) in an endemic area of Santiago del Estero, Argentina. Bull World Health Organ 68: $737-746$.

\section{APPENDIX 1}

Let us call A the event corresponding to the opossum's infection, B the event that the bug "bites", and C the event the bug is ingested, and let us use the symbol ' to indicate their respective complements. We will then have 


$$
\begin{aligned}
A^{\prime}= & \left(A^{\prime} \cap B\right) \cup\left(A^{\prime} \cap B^{\prime}\right) \quad \text { and } \\
P\left(A^{\prime} \mid C\right)= & P\left(A^{\prime} \cap B \mid C\right)+P\left(A^{\prime} \cap B^{\prime} \mid C\right) \\
= & P\left(A^{\prime} \mid B \cap C\right) P(B \mid C) \\
& +P\left(A^{\prime} \mid B^{\prime} \cap C\right) P\left(B^{\prime} \mid C\right)
\end{aligned}
$$

Let us call $p_{1}$ the probability that a bite will infect an opossum, $p_{2}$ the probability that a bug that has been eaten by an opossum will infect it, either because it had bitten the opossum previously, or as a direct result of having been ingested, $p_{3}$ the probability that if a bug is ingested it will infect the opossum exclusively because of the ingestion itself, and $p_{4}$ the probability that an ingested bug might had bitten the opossum before being ingested. Then from Equation 6, it follows that

$$
1-p_{2}=\left(1-p_{1}\right)\left(1-p_{3}\right) p_{4}+\left(1-p_{3}\right)\left(1-p_{4}\right)
$$

from where we can solve for $p_{3}$.

$$
p_{3}=1-\frac{\left(1-p_{2}\right)}{\left(1-p_{1} p_{4}\right)}=\frac{p_{2}-p_{1} p_{4}}{1-p_{1} p_{4}}
$$

For the calculation of $p_{6}$, let us call $A_{1}$ the event that infection due to a bite occurs, $A_{2}$ the event that infection due to predation of one triatomine occurs, and $A$ the event that an infection of the opossum occurs. Then

$$
P\left(A^{\prime}\right)=P\left(A_{1}{ }^{\prime}\right) P\left(A_{2}{ }^{\prime}\right)=\left[1-P\left(A_{1}\right)\right]\left[1-P\left(A_{2}\right)\right]
$$

and since $P\left(A_{1}\right)=p_{1} p_{4}$ and $P\left(A_{2}\right)=p_{3} p_{5}$ then

$$
p_{6}=1-\left(1-p_{1} p_{4}{ }^{\prime}\right)\left(1-p_{3} p_{5}\right)
$$

\section{APPENDIX 2}

Under the hypothesis iii that the probability that an ingested bug had bitten the opossum previously is the same as the probability of "biting" an opossum in the event that the bug had not been ingested, we have to estimate simultaneously $p_{1}, p_{2}$, and $p_{4}$ by maximum likelihood. The joint probability function of $(Y, X)$ conditional on $W$ is given by

$$
\begin{aligned}
& P\left(X=x, Y=y \mid W=w, p_{1}, p_{3}, p_{4}\right) \\
&= P\left(X=x, W=w, p_{4}\right) \\
& \quad \times P\left(Y=y \mid W=w, X=x, p_{1}, p_{3}, p_{4}\right)
\end{aligned}
$$

As the distribution of $X$ given $W$ is binomial with probability $p_{4}$ and $(k-w)$ trials, we have

$$
P\left(X=w \mid X=w, p_{4}\right)=\left(\begin{array}{c}
k-w \\
x
\end{array}\right) p_{4}{ }^{x}\left(1-p_{4}\right)^{n-w-k}
$$

On the other hand as $P\left(Y=y \mid W=w, X=x, p_{1}, p_{3}, p_{4}\right)$ is given by Equations 1 and 2, where $p_{2}$ should be expressed in terms of $p_{1}, p_{3}$, and $p_{4}$ solving from Equation 3 , it results

$$
p_{2}=1-\left(1-p_{1}\right)\left(1-p_{3}\right) p_{4}-\left(1-p_{3}\right)\left(1-p_{4}\right)
$$

We can now calculate the joint likelihood function of the values $\left(x_{1}, y_{1}\right), \ldots,\left(x_{n}, y_{n}\right)$ conditional to $W_{1}=w_{1}, \ldots, W_{n}$ $=w_{n}$.

$$
\begin{aligned}
& L\left(p_{1}, p_{3}, p_{4}\right) \\
& \quad=\prod_{i=1}^{n} P\left(Y=y_{i}, X=x_{i} \mid W=w_{i}, p_{1}, p_{3}, p_{4}\right)
\end{aligned}
$$

Maximizing $L^{*}\left(p_{1}, p_{3}, p_{4}\right)$ we can estimate simultaneously $p_{1}$, $p_{3}$ and $p_{4}$

\section{APPENDIX 3}

The confidence intervals of $p_{1}, p_{2}$ and $p_{3}$ were obtained using the likelihood ratio statistic. If $\hat{p}_{1}$ and $\hat{p}_{2}$ are the maximum likelihood estimators of $p_{1}$ and $p_{2}$, respectively, that is, the values that maximize $L\left(p_{1}, p_{2}\right)$, and if $\hat{p}_{2}(p)$ is the maximum likelihood estimators of $p_{2}$ assuming $p_{1}=p$, then the likelihood ratio statistic is given by

$$
R(p)=2 \log \left(\frac{L\left(\hat{p}_{1}, \hat{p}_{2}\right)}{L\left(p, \hat{p}_{2}(p)\right)}\right)
$$

Let $Z_{\alpha}\left(p_{1}, p_{2}\right)$ be the $\alpha$ quantile of $R(p)$ conditional to $W_{1}=$ $w_{1}, \ldots, W_{n}=w_{n}$, when the true probabilities are $p_{1}$ and $p_{2}$, that is

$$
\begin{gathered}
P\left(R\left(p_{1}\right) \leq Z_{\mathrm{\alpha}}\left(p_{1}, p_{2}\right) \mid X_{1}=x_{1}, \ldots, X_{n}=x_{n},\right. \\
\left.W_{1}=w_{1}, \ldots, W_{n}=w_{n}, p_{1}, p_{2}\right)=\alpha
\end{gathered}
$$

And let us define

$$
Z_{\alpha}^{*}\left(p_{1}\right)=\max _{p_{2}} Z_{\alpha}\left(p_{1}, p_{2}\right)
$$

An exact confidence interval of level $(\alpha-1)$ for $p_{1}$ is defined by

$$
Z_{\alpha}^{*}\left(p_{1}\right)=\max _{p_{2}} Z_{\alpha}^{*}\left(p_{1}, p_{2}\right)
$$

An approximate confidence interval of level $(\alpha-1)$ for $p_{1}$ is defined by

$$
I_{1}=\left\{p: R(p) \leq Z_{1-\alpha}{ }^{*}(p)\right\}
$$

For large $n, Z_{\alpha}\left(p_{1}, p_{2}\right)$ and $Z_{\alpha}{ }^{*}\left(p_{1}\right)$ are approximately the $\alpha$ Quantile of the $\chi^{2}$ distribution with $\chi_{\alpha, 1}^{2}$ degrees of freedom. The exact confidence intervals are difficult to calculate for they require knowledge about the exact percentiles $Z_{(1-\alpha)}\left(p_{1}, p_{2}\right)$.

\section{APPENDIX 4}

We assume that the time between triatomines "bites" and the time between the ingestion of one triatomine and the next by an opossum are typical waiting times with no memory; that is, we accept that the probability of the next bite and of the next ingestion are independent of when each of those events has occurred previously. Then the waiting times between "biting" and ingestions are well described by an exponential distribution. The average "biting" time $\left(\lambda_{x}\right)$ and the average ingestion time $\left(\lambda_{w}\right)$ were obtained solving the equations

$$
\begin{aligned}
& P\left(T<T_{e}\right)=1-e^{-T_{e} \lambda_{x}}=p_{4}{ }^{\prime} \\
& P\left(T<T_{e}\right)=1-e^{-T_{e} \lambda_{w}}=p_{5}
\end{aligned}
$$

where $T_{e}$ is the time an opossum-triatomine encounter lasts. The $p^{\prime}{ }_{4}$ and $p_{5}$ for encounter times between opossums and triatomines are as in the laboratory experiments, we replace in (A4-3) and (A4-4) $T_{e}$ by $23 \mathrm{hr}$ :

$$
\begin{gathered}
p_{4}{ }^{\prime}=1-e^{-23 \lambda_{x}} \\
p_{5}=1-e^{-23 \lambda_{w}}
\end{gathered}
$$


We also assume that the number of encounters between a triatomine and an opossum, during the entire life span of the latter, is a random process, that is, it follows a Poisson distribution with average $\mu$. Let us call $Z$ the number of encounters with a Poisson of mean $\mu$; then

$$
P(Z=x)=\frac{\mu^{z} e^{-\mu}}{z !}
$$

If we define $\mathrm{A}$ as the event that an opossum becomes infected during any period in its life, then

$$
P\left(A^{\prime}\right)=\sum_{z=0}^{\infty} P\left(A^{\prime} \mid Z=z\right) P(Z=z)
$$

and as

$$
P\left(A^{\prime} \mid Z=z\right)=\left(1-p_{6}\right)^{z}
$$

by replacing (A4-5) and (A4-7) in (A4-6) we obtain

$$
\begin{aligned}
P\left(A^{\prime}\right) & =\sum_{z=0}^{\infty}\left(1-p_{6}\right)^{z} \frac{\mu^{z} e^{-\mu}}{z !}=\sum_{z=0}^{\infty} \frac{\left[\mu\left(1-p_{6}\right)\right]^{z} e^{-\mu}}{z !} \\
& =e^{\left(1-p_{6}\right) \mu} e^{-\mu}=e^{-\mu p_{6}}
\end{aligned}
$$

Then, the probability that an opossum becomes infected during its life is $\mathrm{P}(\mathrm{A})$ (which we will call $p_{7}$ ) and is given by

$$
p_{7}=1-e^{-\mu p_{6}}
$$

Solving for $\mu$ we have

$$
\mu=-\frac{\ln \left(1-p_{7}\right)}{p_{6}}
$$

In the case of an encounter lasting $20 \mathrm{hr}$, applying equations (A4-3) and (A4-4) we obtain $p_{4}^{\prime}=0.612$ and $p_{5}=0.217$, which when inserted into equation (6) produces $p_{6}=0.054$. If $p_{7}$ as an estimate of the field prevalence is, say, 0.35 , then $\mu=7.95$. 